\title{
Carbonic anhydrase IX antigen differentiates between preneoplastic malignant lesions in non-small cell lung carcinoma
}

\author{
P. Vermylen****, C. Roufosse ${ }^{+}$, A. Burny ${ }^{\#}$, A. Verhest ${ }^{+}$, T. Bosschaerts ${ }^{\S}$, \\ S. Pastorekova ${ }^{++}$, V. Ninane ${ }^{* *}$, J-P. Sculier*
}

Carbonic anhydrase IX antigen differentiates between preneoplastic malignant lesions in non-small cell lung carcinoma. P. Vermylen, C. Roufosse, A. Burny, A. Verhest, T. Bosschaerts, S. Pastorekova, V. Ninane, J-P. Sculier. (C)ERS Journals Ltd 1999.

ABSTRACT: The MaTu interval (MN)/carbonic anhydrase (CA) IX tumourassociated antigen is a protein that is normally expressed in the gut and belongs to the carbonic anhydrase enzyme family (CA IX). It has been detected in tumour cell lines and in some solid tumours including cervical, oesophageal and clear cell renal carcinoma. This study determined MN/CA IX expression in 65 primary non-small cell lung cancer resected with curative intent and in $\mathbf{3 8}$ bronchial preneoplastic lesions, carcinoma in situ or microinvasive carcinoma as well as in normal bronchial tissue.

The presence of MN/CA IX was detected using immunohistochemistry and Western blot analysis, whenever frozen material was available.

Immunostaining was positive in $52 / 65(80 \%)$ of the tumour samples. The staining was more often focal than diffuse. The percentage of stained cells in positive tumours was highly variable, ranging $1-85 \%$. The pattern of immunostaining was predominantly cytoplasmic with a membranous reinforcement $(87 \%)$. The intensity was mainly strong $(69 \%)$. The presence of the protein in the tumour was confirmed by Western blot analysis in the eight samples tested. All the morphologically normal epithelia, except in close vicinity of tumours in some cases, as well as the preneoplastic bronchial lesions (basal cell hyperplasia, metaplasia and dysplasia) were immunonegative for MN/CA IX expression. In contrast, carcinoma in situ and microinvasive epithelioma showed the presence of MN-immunopositive tumoural cells in 5/7 and 4/5 of the samples, respectively.

These data suggest that MN/CA IX is a useful marker for the differentiation between preneoplastic lesions and bronchial non-small cell lung cancer in the lung. Eur Respir J 1999; 14: 806-811.

\author{
* Service de Médecine et Laboratoire d'In- \\ vestigation Clinique et d'Oncologie Ex- \\ périmentale H.J. Tagnon, **Dept of Chest \\ Medicine and ${ }^{5}$ Dept of Thoracic Surgery, \\ Centre Hospitalo-universitaire Saint-Pierre; \\ ${ }^{+}$Dept of Pathology and ${ }^{\#}$ Dept of Molecular \\ Biology, Institut Jules Bordet, Centre des \\ Tumeurs de l'Université Libre de Bruxelles, \\ Belgium; ${ }^{++}$Institute of Virology, Slovak \\ Academy of Sciences, Bratislava, Slovak \\ Republic. \\ Correspondence: J-P. Sculier Service de \\ Médecine Institut Jules Bordet 1, rue \\ Héger-Bordet B-1000 Brussels Belgium \\ Fax: 3225343756 \\ Keywords: Carbonic anhydrase \\ lung cancer biology \\ MN-CA IX antigen \\ preneoplastic bronchial lesions \\ tumour marker \\ Received: February 111999 \\ Accepted after revision June 221999 \\ This work was supported by the FNRS \\ (grants 9.4587 .95 and 3.4505.98), Télévie \\ fellowships (n7.4523.96 and n7.4512.98), \\ the Vesale Foundation (grants UP 1996, P. \\ Vermylen; and 1997, C. Roufosse) and the \\ Lambeau-Marteaux Foundation.
}

In industrialized countries, lung cancer is the leading cause of death, and its high mortality is mainly attributed to late diagnosis. The disease develops insidiously, with symptoms that usually occur when the tumour has reached a size or degree of local invasion that compromises curative surgical resection. Recent efforts have been performed to understand the natural history of lung cancer, namely by the study of preneoplastic lesions [1] that can now be better detected by new techniques with high sensitivity such as fluorescence bronchoscopy [2].

The recognition of multiple morphological and biological alterations in the epithelium of patients at a high risk of developing lung cancer has lead to an intensive search for new biomarkers, that would be specifically associated with malignant transformation. The detection of these biomarkers in sputum or biopsies would possibly allow an earlier diagnosis.

Recently, a tumour-associated antigen, called MaTu (MN) or carbonic anhydrase IX (CA IX)), has been described in the highly malignant cervical HeLa cell line [3]. The MN/CA IX antigen is an $N$-glycosylated protein detected as two distinct protein bands of 54 and $58 \mathrm{kDa}$ on Western blots, probably due to the posttranslational processing of a single gene product. The expression of
$\mathrm{MN}$ in normal tissues seems to be confined to the gastrointestinal tract. It is found in the basolateral surfaces of the gastric mucosal cells and of enterocytes of the small and large intestine. In the small intestine, its expression is particularly intense in the duodenum and jejunum in the crypts. Its expression is weaker in the colon [4]. Thus, the presence of $\mathrm{MN}$ appears to correlate with the rapidity of cell proliferation in the normal gut.

MN/CA IX has also been found in various solid tumours developed from tissues that normally do not express it. It was first described in cervical dysplasia, cervical intraepithelial neoplasia (CIN) as well as in invasive squamous and glandular neoplasms of the uterine cervix [5-7]. The MN/ CA IX protein has also been detected in preneoplastic lesions, squamous cell carcinomas and adenocarcinomas of the oesophagus [8]. Finally, it is expressed in clear cell carcinomas of the kidney, while it is not detectable in normal kidney and in benign renal lesions $[9,10]$.

The presence of $\mathrm{MN}$ in lung cancer has not been studied to date. The present report is an investigation of MN/CA IX expression in primary non-small cell lung cancer (NSCLC) resected with curative intent, in bronchial preneoplastic lesions and early lung cancer (carcinoma in situ (CIS) and microinvasive carcinoma). 


\section{Materials and methods}

\section{Tissue specimens}

Tissue samples were obtained from 65 resected invasive NSCLC and from preneoplastic and early lung cancer lesions as well as normal mucosa obtained by bronchial biopsies in patients at high risk for lung cancer, using fluorescence bronchoscopy. The 65 resected tumours were obtained from the Pathology Department; they included 31 adenocarcinomas, five bronchio-alveolar carcinomas, 24 squamous cell carcinomas, four adenosquamous carcinomas and one undifferentiated large cell carcinoma. Normal bronchial $(n=34)$ and alveolar $(n=42)$ epithelium in close contact to the tumour as well as normal matched lung tissues were also assessed. Preneoplastic and early lung cancer lesions as well as normal mucosa samples were obtained by bronchial biopsies in 36 patients at high risk for lung cancer, using fluorescence bronchoscopy. The samples were chosen as representative of particular morphological preneoplastic alterations, including normal epithelium $(n=10)$, basal cell hyperplasia $(n=8)$, metaplasia $(n=10)$, dysplasia $(n=8)$, CIS $(n=7)$ and micro-invasive tumours $(n=5)$.

All tissues were fixed in 10\% neutral buffered formalin within a few hours following the surgical resection. In available cases, fresh tissue was snap-frozen in liquid nitrogen and stored at $-80^{\circ} \mathrm{C}$ until processing. Histology was classified according to the new World Health Organisation (WHO) 1998 classification [11], based on routine haematoxylin-eosin staining, combined with periodic acid Schiff staining and immunohistochemistry when necessary. Bronchial biopsies were classified as normal epithelium, basal cell hyperplasia, metaplasia, dysplasia, CIS or microinvasive epithelioma. Morphological staging was performed blindly by two of the authors (C. Roufosse and P. Vermylen).

\section{Immunohistochemical studies}

Immunohistochemistry was performed according to a standard peroxidases technique with the murine monoclonal antibody M75 (kindly provided by J. Pastorek, Institute of Virology, Slovak Republic). Five-micron thick sections of paraffin-embedded tissues were deparaffinized. The endogenous peroxidases were quenched with a solution of $0.3 \%$ hydrogen peroxide in methanol for $30 \mathrm{~min}$. The slides were submitted to antigen retrieval in citric acid monohydrate $0.01 \mathrm{M}(\mathrm{pH} 6.0$ ) consisting of three 5-min of microwave treatments at $800 \mathrm{~W}$ [5]. Pre-incubation with blocking serum (bovine serum albumin $1.5 \%$ in trishydroxymethyl-amino methane (Tris) buffer, $\mathrm{pH}$ 7.5) for 20 min enabled the specificity of the immunostaining to be increased by lowering non-specific background according to the method of LiaO et al. [5]. Contact with the primary monoclonal antibody $\mathrm{M} 75$, diluted 1:5,000 in Tris- $\mathrm{HCl}$ was performed at room temperature for $60 \mathrm{~min}$, followed by $30 \mathrm{~min}$ incubation with a secondary biotinylated anti-mouse immunoglobulin G antibody ("Super Sensitive" kit; Biogenex, San Ramon, CA, USA), before being exposed to horseradish peroxidase-conjugated streptavidine ("Super Sensitive" kit; Biogenex) for 30 min. The slides were then stained with diaminobenzidine tetrahydrochloride (Dako, Carpinteria, CA, USA). They were counterstained with haematoxylin and mounted with permount. All the incubations were performed at room temperature, in humidified chambers. The slides were rinsed twice in Tris buffer for $10 \mathrm{~min}$ between each incubation. Positive controls consisted of HeLa cells while negative controls were performed by omitting the primary M75 antibody. Immunostaining was semi-quantitatively scored as the percentage of positive tumour cells in the total field of a single section. The pattern of staining was qualified as cytoplasmic (C), cytoplasmic with a membranous reinforcement $(\mathrm{CM})$ or membranous only (M). The intensity of the staining was recorded as absent, weak or strong.

\section{Western blot analysis of $M N$ expression}

Total protein content was extracted from the frozen tissue samples after homogenization on ice in a buffer containing Phenyl methyl sulphonyl fluoride, in $10 \mathrm{mM}$ ethylene diamine tetra-acetic acid (EDTA) (RIPA) buffer solution containing a broad spectrum of protease inhibitors (phosphate-buffered saline (PBS) $\mathrm{pH} 7.2,1 \%$ Triton X$100,0.1 \%$ sodium deoxycholate and $10 \mu \mathrm{g} \cdot \mathrm{mL}^{-1}$ phenylmethylsulfonyl fluoride). Snap-frozen samples of gastric mucosa were used as positive controls. Protein quantification was performed according to the Lowry method using the Biorad protein assay (Biorad Laboratories, Hercules, CA, USA) assay. A 40- $\mu$ g sample of each protein extract was separated by electrophoresis on a $12 \%$ sodium dodecyl sulphate (SDS) (Prosieve 50; Sanvertech, Boechout, the Netherlands) polyacrylamide electrophoresis gel, then transferred to a nitrocellulose membrane (polyvinylidene fluoride (PVDF)) in buffer (Tris $25 \mathrm{mM}$, glycine $190 \mathrm{mM}$, methanol 20\%). The membrane was blocked for $60 \mathrm{~min}$ at room temperature in TBST buffer (Tris- $\mathrm{HCl} 10 \mathrm{mM}, \mathrm{NaCl}$ $150 \mathrm{mM}$, Tween-20 0.1\%) and 5\% dry milk. The blot was then incubated overnight at $4{ }^{\circ} \mathrm{C}$ with the murine monoclonal antibody M75, diluted 1:3,000 in TBST. The membrane was incubated with a secondary peroxidase-bound goat antimouse antibody diluted 1:2,500 in TBST (Dako) for $60 \mathrm{~min}$ at room temperature. The protein bands were then revealed with a sensitive chemiluminescent detection system (ECLplus; Amersham, Arlington Heights, IL, USA).

\section{Statistical analysis}

Comparisons of the distributions were made by using nonparametric tests (Kruskal-Wallis and Mann-Whitney tests). A p-value $<0.05$ was considered to be statistically significant.

\section{Results}

Tumour specimens included 31 adenocarcinoma, five bronchioloalveolar carcinoma, 24 squamous cell carcinoma, four adenosquamous carcinoma and one undifferentiated NSCLC. Normal bronchial $(n=34)$ and alveolar $(n=42)$ epithelium in close contact to the tumour was assessed as well as normal matched lung tissue. Bronchial biopsies were obtained in 24 patients at high risk for lung cancer and who underwent fluorescence bronchoscopy. The examination was performed for preoperative assessment of a synchronous lung cancer in four patients, or of a synchronous head and neck tumour in three, or for 
Table 1. - MN expression in resected non-small cell lung cancer (NSCLC)

\begin{tabular}{|c|c|c|c|c|c|c|}
\hline & \multicolumn{6}{|c|}{ Histological type } \\
\hline & Adenoc & BAC & Squam & Adenosq & Undiff & Total \\
\hline Patients $\mathrm{n}$ & 31 & 5 & 24 & 4 & 1 & 65 \\
\hline \multicolumn{7}{|c|}{ Percentage of cells immunostained } \\
\hline Negative $(0 \%)$ & 6 & 3 & 1 & 2 & 1 & 13 \\
\hline Positive $(1-100 \%)$ & 25 & 2 & 23 & 2 & 0 & 52 \\
\hline $1-10 \%$ & 12 & 1 & 9 & 1 & 0 & 23 \\
\hline $11-20 \%$ & 2 & 0 & 1 & 0 & 0 & 3 \\
\hline $21-40 \%$ & 3 & 0 & 4 & 0 & 0 & 7 \\
\hline $41-60 \%$ & 6 & 1 & 4 & 1 & 0 & 12 \\
\hline $61-80 \%$ & 2 & 0 & 3 & 0 & 0 & 5 \\
\hline $81-100 \%$ & 0 & 0 & 2 & 0 & 0 & 2 \\
\hline \multicolumn{7}{|c|}{ Immunostaining pattern } \\
\hline Cytoplasmic & 0 & 2 & 2 & 0 & 0 & 4 \\
\hline Membranous & 3 & 0 & 0 & 0 & 0 & 3 \\
\hline Both & 22 & 0 & 21 & 2 & 0 & 45 \\
\hline \multicolumn{7}{|c|}{ Immunostaining intensity } \\
\hline Absent & 6 & 3 & 1 & 2 & 1 & 13 \\
\hline Weak & 7 & 1 & 8 & 0 & 0 & 16 \\
\hline Strong & 18 & 1 & 15 & 2 & 0 & 36 \\
\hline
\end{tabular}

Adenoc: adenocarcinoma; BAC: bronchiolo-alveolar carcinoma; Squam: squamous cell carcinoma; Adenosq: adenosquamous carcinoma; Undiff: undifferentiated NSCLC.

screening of lung cancer in nine patients with previous history of lung cancer and in eight with severe tobacco exposure. They included morphologically normal epithelium $(n=10)$, basal cell hyperplasia $(n=8)$, metaplasia $(n=$ $10)$, dysplasia $(\mathrm{n}=8), \mathrm{CIS}(\mathrm{n}=7)$ and microinvasive tumours $(\mathrm{n}=5)$.

Immunohistochemical MN antigen expression in invasive tumours

The results are shown in table 1. Immunostaining was positive in $80 \%$ of the tumour samples $(52 / 65)$. The staining (fig. 1) was more often focal than diffuse, the positive cells being isolated or in islands. The percentage of stained cells in positive tumours was highly variable, ranging $1-85 \%$ (mean \pm SD $25 \pm 25 \%)$. The pattern of immunostaining was predominantly cytoplasmic with a membranous reinforcement $(45 / 52 ; 87 \%)$. The intensity was mainly strong $(69 \%)$. Considering the percentage of

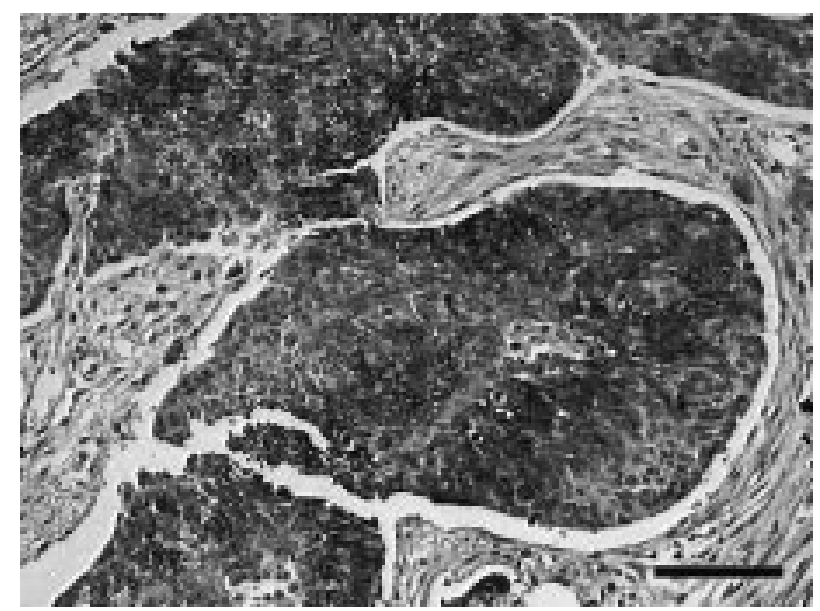

Fig. 1. - Squamous cell carcinoma with a positive immunostaining for $\mathrm{MN}$ with a cytoplasmic-membranous pattern. (Internal scale bar $=25 \mu \mathrm{m}$.) positive cells, intensity and pattern of staining, there were no significant difference between squamous cell and adenocarcinoma histological subtypes (Kruskal-Wallis test; $\mathrm{MN}$ frequency, $\mathrm{p}=0.122 ; \mathrm{MN}$ intensity, $\mathrm{p}=0.449$; MN staining pattern, $\mathrm{p}=0.609$ ). There was no correlation with tumour differentiation either (Kruskal-Wallis test; $\mathrm{MN}$ frequency, $\mathrm{p}=0.0792 ; \mathrm{MN}$ intensity, $\mathrm{p}=0.225 ; \mathrm{MN}$ staining pattern, $\mathrm{p}=0.404$ ).

"Normal" bronchial and alveolar epithelium in close vicinity to the tumours had cells with immunopositive staining in 14/34 bronchial samples (41\%) and in 3/42 alveolar samples (7\%). All matched normal lung specimens sampled at a distance from the tumour were immunonegative.

\section{Western blot analysis of MN expression}

A series of eight frozen tumour specimens, with a matched sample of normal lung tissue taken at a distance from the tumour and in the same patient, were examined by Western blot. The eight tumour specimens contained the 54-58 $\mathrm{kDa}$ bands, recognized by the murine M75

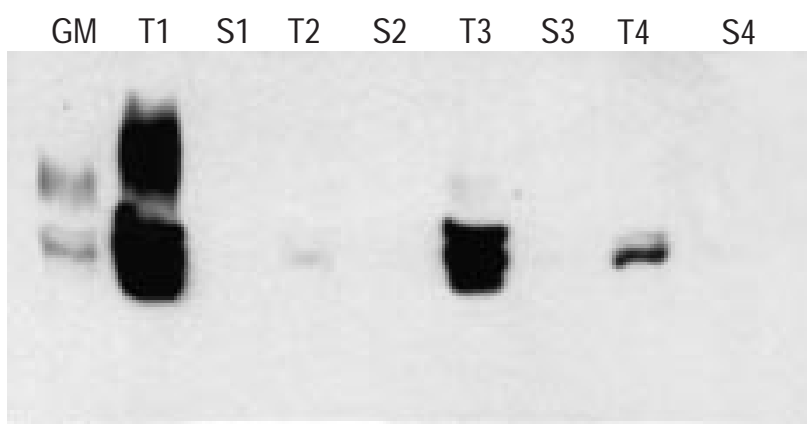

Fig. 2. - Western blot analysis of a squamous cell carcinoma showing the $54-58 \mathrm{kDa}$ bands recognized as MN by the murine M75 monoclonal antibody, gastric mucosa (GM) being used as the positive in four matched samples of tumour (T) and normal tissues (S). 
monoclonal antibody, and thus indentifying the presence of MN protein (fig. 2). All the eight control lung tissue specimens were negative.

\section{Immunohistochemical MN antigen expression in bron- chial biopsies}

The results are shown in table 2. All the morphologically normal epithelia as well as samples of basal cell hyperplasia, metaplasia and dysplasia were immunonegative for MN/CA IX expression. In contrast, CIS and microinvasive epitheliomas showed the presence of MNimmunopositive tumoural cells in $5 / 7$ and $4 / 5$ of the samples, respectively. This immunostaining was predominantly weak and cytoplasmic in CIS (fig. 3) while it was more intense and most often cytomembranous in microinvasive lesions (fig. 4). Western blot analysis of $\mathrm{MN}$ expression in CIS was not performed because the size of the lesions was too small to obtain adequate material to perform the test. Figure 5 provides a graphical summary of the main study results.

\section{Discussion}

This study suggests that MN/CA IX is a biomarker of potential interest for lung cancer because it appears to be expressed when a bronchial lesion becomes neoplastic. Indeed, immunostaining is negative in normal epithelium (except when it is taken from close vicinity to tumours, in some cases) and in precancerous stages and, in contrast, is positive in a high percentage of CIS, microinvasive epithelioma and various histological subtypes of resected NSCLC.

MN belongs to the family of the CAs. These proteins are zinc metallo-enzymes that catalyse the interconversion of carbonic acid to carbon dioxide, thus participating in a wide

Table 2. - MN expression in preneoplastic and early neoplastic bronchial lesions

\begin{tabular}{lcc}
\hline & \multicolumn{2}{c}{ Histological lesion } \\
\cline { 2 - 3 } & CIS & $\begin{array}{c}\text { Microinvasive } \\
\text { epithelioma }\end{array}$ \\
\hline Patients n & 7 & 5 \\
Percentage of cells immunostained & & \\
Negative (0\%) & 2 & 1 \\
Positive (1-100\%) & 5 & 4 \\
$1-20 \%$ & 5 & 3 \\
$21-40 \%$ & 0 & 1 \\
$41-60 \%$ & 0 & 0 \\
$61-80 \%$ & 0 & 0 \\
$81-100 \%$ & 0 & 0 \\
Immunostaining pattern & 5 & 1 \\
Cytoplasmic & 0 & 0 \\
Membranous & 0 & 3 \\
Both & & \\
Immunostaining intensity & 2 & 1 \\
Absent & 4 & 1 \\
Weak & 1 & 3 \\
Strong & & \\
\hline
\end{tabular}

CIS: carcinoma in situ. All the morphologically normal epithelia $(n=10)$ and samples of basal cell hyperplasia $(n=8)$, metaplasia $(n=10)$ and dysplasia $(n=8)$ were negative for $M N / C R$ IX expression. variety of biological processes $[12,13]$. In addition, the CA domain could possess a catalytically independent function of ligand-binding sites that mediates the signal transduction and influences the cell behaviour. This was proven for the receptor protein tyrosine phosphatase (RPTPb) containing an enzymatically inactive CA domain [14]. When tumour cell line 3T3 from the National Institutes of Health, Bethesda, MD, USA (NIH 3T3) fibroblasts are transfected with a plasmid expressing the MN/CA IX protein, they under-go morphological transformation with all typical features in vitro, including increased proliferation and anchorage independence [3]. On the basis of the expression pattern in normal and cancerous tissues, MN/CA IX is considered to play a potential regulatory role in intercellular communication and cell proliferation, and appears to be involved in oncogenesis.

When initially found in HeLa cells, the MN protein was not known to have a CA domain [5]. Its structure has been recently studied by complementary deoxyribonucleic acid and genomic sequence analysis. It consists of an N-terminal proteoglycan-like region, a central portion with an active enzyme centre that has a high sequence identity to members of the CA family, a transmembrane anchor and a C-terminal intracytoplasmic tail. Correspondingly, the $\mathrm{MN}$ protein has a weak carbonic anhydrase activity, for which reason it is also called CA IX. The membranous reinforcement described in immunohistochemical stainings is in keeping with the presence of a transmembrane anchor.

In humans, it is physiologically expressed in the epithelial cells of the gut with a high proliferation capacity [4]. In colorectal neoplasms, its expression is intense and is correlated with the presence of $\mathrm{Ki}-67$, a well-established marker of cell proliferation [15]. In addition, the presence of MN protein or messenger ribonucleic acid has been documented in cancer arising from tissue that do not normally express this protein, such as the uterine cervix [5] or the kidney [9], suggesting that it may play an important role in the development of solid tumours.

The MN/CA IX protein is not expressed in the normal lung [9] and the present study performed on normal lung by immunochemistry and Western blot analysis confirms the previously published data. It is expressed in CIS but it has not been found in dysplasia, metaplasia and basal cell hyperplasia. This observation is different from what has been documented in cervical cancer [5]. As already reported, $\mathrm{MN}$ is not expressed in the normal cervix. Its expression occurs in the various stages of dysplasia (intraepithelial neoplasia) and it is not specific for cancerassociated human papillomavirus subtypes or of high grade lesions [15]. It is present in $100 \%$ of the cases of invasive squamous cell carcinomas [5] and in $65 \%$ of those of invasive adenocarcinomas [6].

The lack of MN expression in preneoplastic disease of the lung suggests that it would be a very specific marker of the neoplastic phenotype which may help in the differential diagnosis between dysplasia and CIS/microinvasive carcinoma. Indeed, there is so far no specific marker for this purpose since several genetic alterations appear very early in preneoplastic lesions, such as $3 p$ deletion, K-ras activation, $9 p$ deletion or $p 53$ mutation [1]. The data presented here derived from a relatively small cohort are encouraging enough to warrant a large-scale investigation. 
Resected lung cancer, a more advanced disease than CIS or microinvasive radio-occult squamous cell carcinoma, is not invariably associated with $\mathrm{MN}$ expression. In squamous cell carcinoma of the lung, however, $\mathrm{MN}$ appears to be particularly interesting since all but one of the 24 cases studied were found to be immunopositive. Nevertheless, a potential bias due to the limited number of patients, most of which had stage I or II disease, must be taken into account.

a)

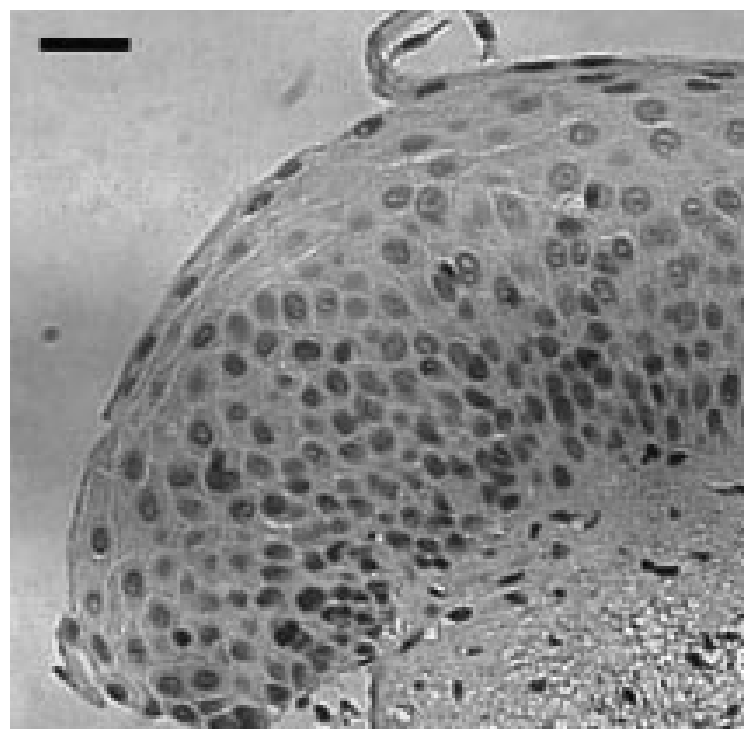

b)

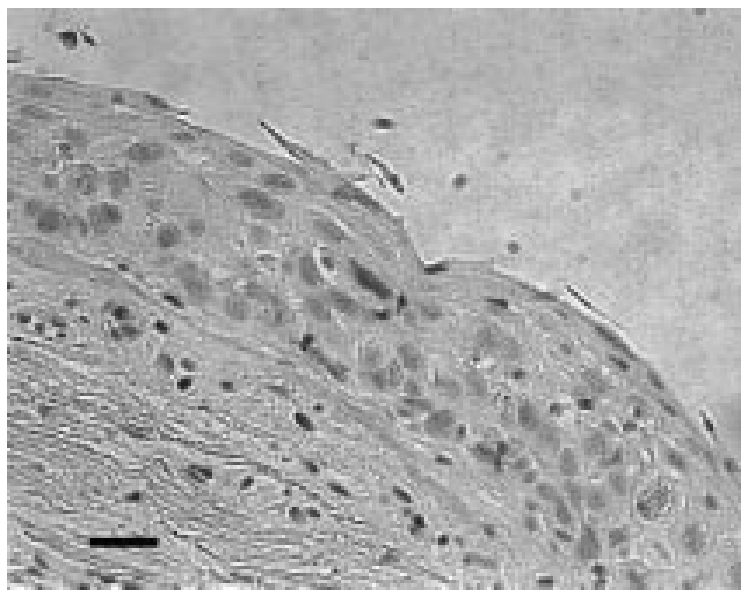

c)

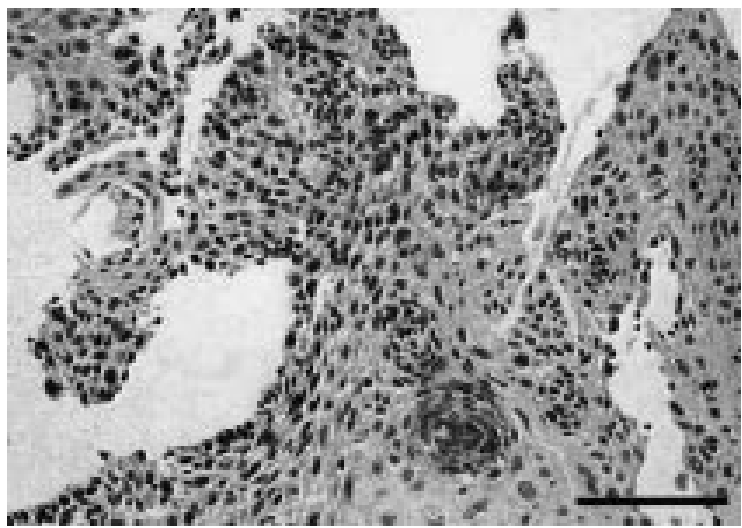

Fig. 3. - Examples of a) metaplasia, b) dysplasia and c) bronchial carcinoma in situ (CIS) with immunostaining for MN, only the CIS being positive with a cytoplasmic pattern. (Internal scale bars $=50 \mu \mathrm{m}$.)
Additional data for locoregionally advanced (stage III) and metastatic (stage IV) tumours are needed. It is indeed possible that MN/CA IX may exert a negative control on cell growth as suggested by a reduction or a loss of its expression in progression of gastric tumour [16], although it may also act as a positive regulator, as observed in other tumours such as colorectal or cervical cancers [5-10, 17, $18]$.

Usually, studies classify samples as either positive or negative for the presence of a marker. MN/CA IX, at least in primary lung tumours, may present with a very heterogeneous repartition, including areas of dense expression and areas of absent or low expression within the same specimen. In these conditions, this study analysed in a semi-quantitative way the MN/CA IX pattern of expression and, for tumours, the complete section sample. This assessment method may be an explanation for the relatively low frequency of immunopositive cells for the tumour as a whole.

In some of the resected NSCLC samples, MN was shown to be expressed in morphologically normal epithelium in close vicinity of the tumour, whereas it was not the case for the normal tissue at distance. The authors have no clear explanation for this observation. A similar observation has been made in the uterine cervix, and has been exploited on cervical smears, where immunopositivity of even normal appearing cells has been found to be predictive of the presence of intraepithelial or invasive carcinoma [19]. It may be speculated that the expression of the MN gene may be induced by the tumour microenvironment [20]. On the other hand, this expression may also reflect a field of cancerization present in the lung, as suggested in a case report [21] by the widespread presence of single somatic $p 53$ mutation in the bronchi. If the theory of field carcinogenesis in the respiratory epithelium is correct, MN could be another example of such diffuse event. However, the absence of its expression in preneoplastic lesions, contrary to mutated $p 53$, may be not in favour of this hypothesis. Further investigations are necessary to clarify this point.

In conclusion, MN/CA IX appears as a potentially useful marker in thoracic oncology to differentiate between preneoplastic bronchial lung lesions and early lung cancer, whether in situ or microinvasive. Expression of $\mathrm{MN}$ in bronchial cells of the sputum should be investigated in

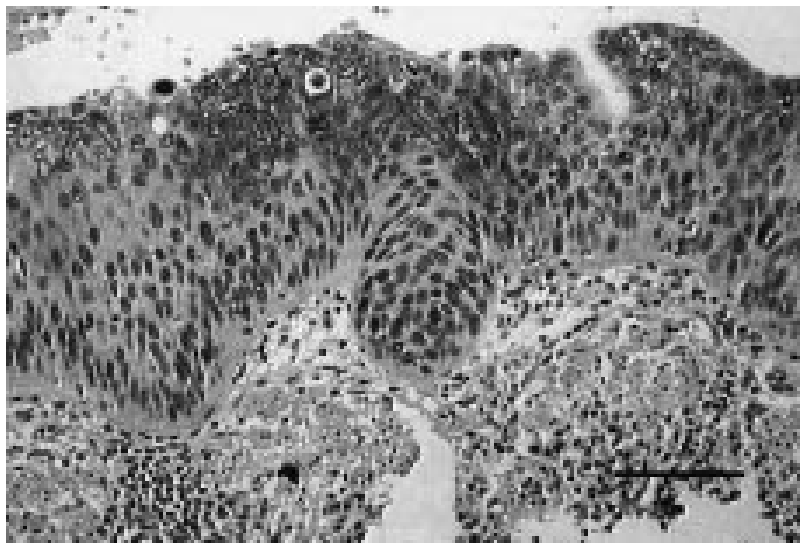

Fig. 4. - Microinvasive squamous cell epithelioma with a strong positive immunostaining for MN with a cytomembranous pattern. (Internal scale bar $=25 \mu \mathrm{m}$.) 


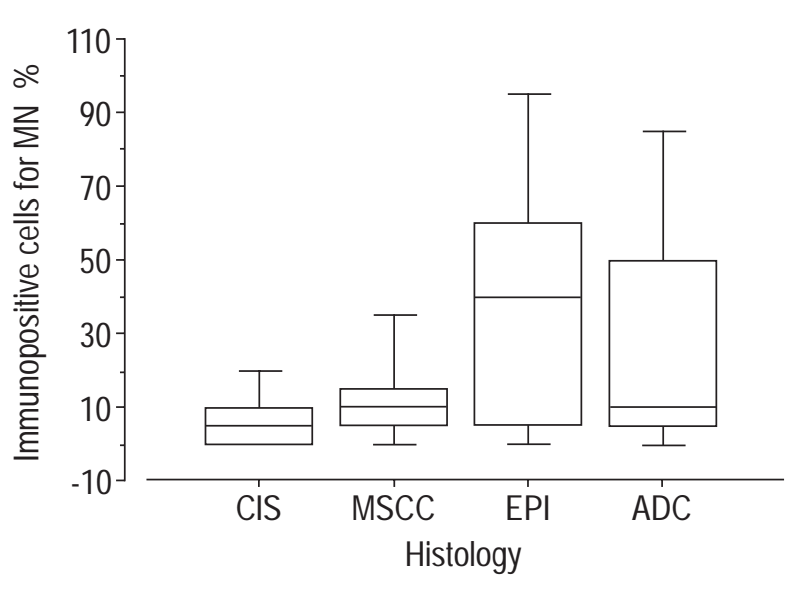

Fig. 5. - Expression of MN (percentage of immunopositive cells) in the main groups studied carcinoma in situ (CIS), microinvasive squamous cell carcinoma (MSCC), invasive squamous cell epithelioma (EPI), adenocarcinoma (ADC). Box and whisker plots with median, interquartile range and minimum and maximum values.

order to assess its potential as a new biological tool for lung cancer screening in high-risk populations. On the other hand, $\mathrm{MN}$ is often present in resected lung cancer but its expression in more advanced tumours has to be analysed in order to determine its potential prognostic value with respect to the survival of the patients or on the response to treatment.

Acknowledgements. The authors would like to thank G. Rouas (Institute Jules Bordet, Brussels, Belgium) and G. Berchem (Centre Hospitalier de Luxembourg, Luxembourg) for their technical help.

\section{References}

1. Vermylen P, Roufosse C, Ninane V, Sculier JP. Biology of pulmonary preneoplastic lesions. Cancer Treat Rev 1997; 23: 241-262.

2. Lam S, Hung JYC, Hung J, LeRiche J, Profio AE, Palcic B. Detection of dysplasia and carcinoma in situ with a lung imaging fluorescence endoscope device. J Thorac Cardiovasc Surg 1993; 105: 1035-1040.

3. Pastorek J, Pastorekkova S, Callebaut I, et al. Cloning and characterization of $\mathrm{MN}$, a human tumour-associated protein with a domain homologous to carbonic anhydrase and a putative helix-loophelix DNA binding segment. Oncogene 1994; 9: 2877-2888.

4. Saarnio J, Parkila S, Kaisa Parkkila A, et al. Immunohistochemistry of carbonic anhydrase isozyme IX (MN/CA IX) in human gut reveals polarized expression in the epithelial cells with the highest proliferative capacity. J Histochem Cytochem 1998; 46: 497-504.

5. Liao SY, Brewer C, Zavada J, et al. Identification of the antigen as a diagnostic biomarker of cervical intraepithelial squamous and glandular neoplasia and cervical carcinomas. Am J Pathol 1994; 145: 598-609.

6. Costa MJ., Ndoye A, Trelford JD. MN protein immu- nolocalization in uterine cervix carcinoma with glandular differentiation. Int J Surg Pathol 1995; 3: 73-82.

7. Brewer C, Liao SY, Wilczynski SP, et al. A study of biomarkers in cervical carcinoma and clinical correlation of the novel biomarker MN. Gyneco Oncol 1996; 63: 337-344.

8. Turner JR, Odze RD, Crum CP, Resnick MB. MN antigen expression in normal, preneoplastic, and neoplastic esophagus: a clinicopathological study of a new cancerassociated biomarker. Hum Pathol 1997; 28: 740-744.

9. McKierman JM, Buttyan R, Bander NH, et al. Expression of the tumour-associated gene $\mathrm{MN}$ : a potential biomarker for human renal cell carcinoma. Cancer Res 1997; 57: 2362-2365.

10. Liao SY, Aurelio ON, Jan K, Zavada J, Stanbridge EJ. Identification of the MN/CA9 protein as a reliable diagnostic biomarker of clear cell carcinoma of the kidney. Cancer Res 1997; 57: 2827-2831.

11. Brambilla E. Anatomo-pathologie: classification et methodologie. In: Le cancer bronchopulmonaire, JP Sculier, V Ninane, JJ Lafitte (eds), Bruxelles, 1998; 15-33.

12. Tashian RE. The carbonic anhydrases: Widening perspectives on their evolution, expression and function. BioEssays 1989; 10: 186-192.

13. Sly WS, Hu PY. Human carbonic anhydrases and carbonic anhydrase deficiencies. Annul Rev Biochem 1995; 64: 375-401.

14. Peles E, Nativ M, Campbell PL, et al. The carbonic anhydrase domain of receptor tyrosine phosphatase is a functional ligand for the axonal cell recognition molecule contactin. Cell 1995; 82: 251-260.

15. Opavsky R, Pastorekova S, Zelnik V, et al. Human MN/ CA9 gene, a novel member of the carbonic anhydrase family: structure and exon to protein domain relationships. Genomics 1996; 33: 480-487.

16. Pastorekova S, Parkkila S, Parkilla AK, et al. Carbonic anhydrase IX, MN/CA IX: analysis of stomach complementary DNA sequence and expression in human and rat alimentary tracts. Gastroenterology 1997; 112: 398-408.

17. Resnick M, Lester S, Tate JE, Sheets EE, Sparks C, Crum CP. Viral and histopathologic correlates of MN and MIB1 expression in cervical intraepithelial neoplasia. Hum Pathol 1996; 27: 234-239.

18. Saarnio J, Parkkila S, Parkilla AK, et al. Immunohistochemical study of colorectal tumours for expression of a novel transmembrane carbonic anhydrase, MN/CA IX, with potential value as a marker of cell proliferation. Am J Pathol 1998; 153: 279-285.

19. Liao SY, Stanbridge EJ. Expression of the MN antigen in cervical Papanicolaou smears is an early diagnostic biomarker of cervical dysplasia. Cancer Epidemiol Biomarkers Prev 1996; 5: 549-557.

20. Cohenford MA, Rigas AB. Cytologically normal cells from neoplastic cervical samples display extensive structural abnormalities on IR spectroscopy: implications for tumour biology. Proc Nay Acad Sci USA 1998; 95: 15327-15332.

21. Franklin WA, Gazdar AF, Haney J, et al. Widely dispersed p53 mutation in respiratory epithelium. J Clin Invest 1997; 100: 2133-2137. 